\title{
Rural health in behavioral medicine: introduction to the special series
}

\author{
M. Bryant Howren ${ }^{1,2}$ (i) $\cdot$ John C. Higginbotham ${ }^{3,4}$
}

Received: 9 June 2021 / Accepted: 11 June 2021 / Published online: 29 June 2021

(C) This is a U.S. government work and not under copyright protection in the U.S.; foreign copyright protection may apply 2021

Keywords Behavioral medicine $\cdot$ Health psychology . Rural health

Approximately 60 million people-or one in five individuals - reside in rural areas throughout the United States (US Census Bureau, 2017). Rural is much more than just a geographical designation but encompasses a host of interrelated social, cultural, and behavioral factors which bear on the health and well-being of its residents. Research consistently indicates that those living in rural areas experience worse health outcomes across numerous diseases and conditions including cancer, depression, diabetes, heart disease, stroke, and suicide (Bolin et al., 2015; Coughlin et al., 2019; Yaemsiri et al., 2019). The reasons for these negative outcomes are many and may include greater travel burden, lack of health insurance, provider shortages, fewer educational resources, limited occupational opportunities, greater poverty, poor infrastructure, social isolation, and sociocultural norms and beliefs that may de-emphasize health-related actions and behaviors in certain contexts such as quitting smoking,

M. Bryant Howren

matthew.howren@med.fsu.edu

1 Department of Behavioral Sciences \& Social Medicine, College of Medicine, Florida State University, Tallahassee, FL, USA

2 Florida Blue Center for Rural Health Research \& Policy, College of Medicine, Florida State University, 1115 W. Call Street, Tallahassee, FL 32306, USA

3 Department of Community and Rural Medicine, College of Community Health Sciences, The University of Alabama, Tuscaloosa, AL, USA

4 The Institute for Rural Health Research, The University of Alabama, Tuscaloosa, AL, USA obtaining vaccinations, or seeking mental health treatment. Collectively, many of these overlapping risks represent what is often referred to as the rural disadvantage. While these disparities and associated explanations have been articulated many times over, researchers and public health practitioners still struggle to understand and resolve these issues.

Importantly, the last decade has seen a marked increase in the attention paid to those in rural and underserved areas. To further understand and address these challenges, it is recognized that more research is needed directly in rural areas and efforts must be focused on increased participation in vital research endeavors. One example is NIH's Precision Medicine Initiative which includes the All of $U s$ Research Program. It aims to recruit one million individuals to establish one of the largest and most diverse health databases in history and will include those from rural, frontier, and underserved areas. Additionally, funding agencies such as the Agency for Healthcare Research \& Quality (AHRQ), Health Resources \& Services Administration (HRSA), National Institutes of Health (NIH), and VA Health Services Research \& Development (HSR\&D) and VA Office of Rural Health (ORH) have increased calls for rural-focused research, recognizing that rural individuals are often less likely to participate in research studies (Tanner et al., 2015) and that increasing levels of participation will require relationship building and outreach perhaps independent of the specific health context under study (Tucker et al., 2016). Much of the evidence-base for all disciplines, including health psychology and behavioral medicine, has come from research conducted in large urban areas; thus, more attention must be paid to examining how behavioral phenomena and their related interventions maintain efficacy and uptake as a function of the unique demographic, social, and cultural milieu in rural areas. The current special series on rural health in the Journal of Behavioral Medicine was meant to 
highlight some of these broader issues and is devoted to rural health research in health psychology and behavioral medicine which is of interest to researchers and clinicians alike.

The special series includes a pair of articles addressing issues in rural cancer survivorship. Bhuiyan et al., (2021) examined age-varying associations between physical activity and psychological outcomes in a sample of 219 rural cancer survivors in Pennsylvania. Participants were survivors of multiple different types of cancer including breast, prostate, and colorectal and completed measures of physical activity and symptoms of depression and anxiety. Analyses utilized intercept-only time-varying effect models (TVEM) to assess the prevalence of symptoms of depression and anxiety as a function of age and to assess the age-varying effects of moderate, vigorous, and total physical activity on symptoms of depression and anxiety. Bhuiyan et al. found that, as compared to older cancer survivors, younger survivors (aged 20-40) experienced a significantly higher prevalence of both depression and anxiety symptoms and advocate for more studies of factors associated with psychological distress in younger adult cancer survivors. Ratcliff et al., (2021) conducted a systematic review of 19 behavioral interventions for rural breast cancer survivors over the last two decades. As noted within the article, compared to urban breast cancer survivors, rural breast cancer survivors experience poorer mental and physical health outcomes and often lack knowledge about post-treatment care. The authors discuss the implications and need for interventions targeting psychosocial support, weight control, and education tailored to rural breast cancer survivors.

Four articles describe interventions for rural military veterans. Bernardy et al., (2021) mailed a direct-to-consumer educational product aimed at decreasing benzodiazepine use in PTSD care to over 1300 veterans in New England, half of which were rural; approximately two-thirds of veterans exhibited a dose reduction with significantly more rural veterans doing so than their urban counterparts. The authors suggest that their approach may have been particularly beneficial for rural veterans in that the educational product left decision-making power to the patient, which may have aligned with many rural individual's strong attitudes toward independence and is an interesting avenue for future research. Dindo et al., (2021) piloted a one-day acceptance and commitment therapy (ACT) group workshop for distressed rural veterans in community-based settings. In order to reach such veterans, the authors partnered with rural veteran-serving nonprofit organizations which often have a high degree of credibility within veteran communities. Results showed improvements in functioning, reintegration, meaning and purpose, as well as reductions in distress three months following workshop participation; the success of the project was a function of fostering collaborations with community-based veterans' organizations, suggesting that community partnerships with rural-serving health clinics and veteran-serving organizations may facilitate identification and engagement of rural veterans in research and care, an area that is receiving increased attention (Howren et al., 2020). O'Hara et al., (2021) conducted a program evaluation of MomMoodBooster, a coachsupported internet-delivered cognitive behavioral therapy (CBT) program for the treatment of maternal depression in veteran women. Program participation was associated with decreased depressive symptoms, increased behavioral activation, and decreased dysfunctional automatic thoughts, with rural women doing as well as urban women in terms of participation and outcomes. Shiner et al., (2021) examined whether the rural-urban disparity in suicide risk among VA users decreased during a time of contemporaneous VA efforts to improve access to and services for mental health care for rural veterans using a retrospective cohort design. They found that the rural-urban suicide disparity persists, but was attenuated after adjustment for age, gender, and race. In particular, rates of suicide were much higher for White as compared to Black veterans, suggesting the need to explore variation in suicide risk by race and rurality.

Lastly, Fischer et al., (2021) conducted a review of sleep health in rural mothers using a social-ecological framework. The authors identified gaps in the literature, social determinants that interact to influence sleep health, and provide recommendations for targeting sleep health in rural mothers. Among other timely recommendations, they suggest incorporating brief and adaptable behavioral sleep interventions with flexible modes of delivery as well as increased screening for behavioral health problems in rural primary care. Research in sleep health continues to grow yet issues specific to rural areas have yet to be fully explored.

These papers represent only a tiny cross-section of the promising work being conducted in the area of rural health in health psychology and behavioral medicine. The intended scope of this series was broad but several important areas are not represented. These include rural care coordination efforts, prevention studies, initiatives to develop/advance population health strategies for rural behavioral health, moderators of treatment impact (e.g., patient, provider, and/or sociocultural factors), emerging or existing areas and populations deserving of greater attention in rural settings (e.g., migrant farm workers, indigenous peoples, persons with HIV, testing and vaccination for COVID-19), and sociocultural considerations related to rural populations in this context to name but a few. We also had hoped to see more research focused on translation and adaptation of existing evidence-based interventions into rural settings through community partnerships, perhaps using informal or paraprofessional delivery mechanisms to engage those hardest to reach. Moreover, as rural areas continue to become more diverse, more research must be also be conducted in 
underrepresented rural minority groups who disproportionately suffer from poverty and other socioeconomic impacts (Bolin et al., 2015) as well as understanding the challenges of recruiting and enrolling all rural residents into behavioral medicine research. The COVID-19 pandemic has exacerbated existing inequalities and has underscored the need to consider these issues in all behavioral medicine research. Lastly, much research focuses on outcomes and content areas in which rural residents fare worse, for good reason, as considerable existing evidence already demonstrates. However, work is needed which highlights things that rural does well, including study of protective factors and related assets and opportunities associated with living in a rural community. This will require a reframing to some degree away from de facto rural-urban comparisons and looking at pockets within rural which have found success and are doing as well or better than comparable rural areas and/or their urban counterparts.

In sum, several diverse patient populations and topic areas are represented in this series, but many other important areas are not, in part due to the nature of an open request for submissions. It is hoped that the included articles are timely, useful, and facilitate even more high quality, methodologically rigorous rural health research in health psychology and behavioral medicine. As rural health continues to rightfully take its place "centerstage" across many health-related disciplines, there will be increased opportunities to undertake impactful behavioral medicine research in this space.

\section{References}

Bernardy, N. C., Montano, M., Cuccurullo, L. A., Breen, K., and Cole, B. F. (2021). Taking the message to the rural patient: Evidencebased PTSD care. [In special series].

Bhuiyan, N., Linden-Carmichael, A. N., Lanza, S. T., Schmitz, K. H., \& Mama, S. K. (2021). Age-varying associations between physical activity and psychological outcomes among rural cancer survivors. [In special series].

Bolin, J. N., Bellamy, G. R., Ferdinand, A. O., Vuong, A. M., Kash, B. A., Schulze, A., \& Helduser, J. W. (2015). Rural healthy people
2020: New decade, same challenges. Journal of Rural Health, 31, 326-333.

Coughlin, S. S., Clary, C., Johnson, J. A., Berman, A., Heboyan, V., Benevides, T., Moore, J., \& George, V. (2019). Continuing challenges in rural health in the United States. Journal of Environment and Health Sciences, 5, 90-92.

Dindo L, Roddy M, Boykins D, et al. (2021). Combination outreach and wellness intervention for distressed rural veterans: Results of a multimethod pilot study. [In special series].

Fischer AR, Green SM, Gunn HE. (2021). Social-ecological considerations for the sleep health of rural mothers. [In special series].

Howren, M. B., Kazmerzak, D., Kemp, R. W., Boesen, T. J., Capra, G., \& Abrams, T. E. (2020). Identification of military veterans upon implementation of a standardized screening process in a federally qualified health center. Journal of Community Health, $45,465-468$.

Ratcliff, C. G., Torres, D., Tullos, E. A., Geng, Y., \& Lu, Q (2021). A systematic review of behavioral interventions for rural breast cancer survivors. [In special series].

Shiner, B., Teltzman, T., Cornelius, S. L., Gui, J., Forehand, J., and Watts, B. V. (2021). Recent trends in rural-urban suicide disparity among veterans using VA health care. [In special series].

Solness CL, Kroska EB, Holdefer PJ, O'Hara MW. (2021). Treating postpartum depression in rural veterans using internet-delivered CBT: Program evaluation of MomMoodBooster. [In special series].

Tanner, A., Kim, S. H., Friedman, D. B., Foster, C., \& Bergeron, C. D. (2015). Barriers to medical research participation as perceived by clinical trial investigators: Communicating with rural and African American communities. Journal of Health Communication, 20, 88-96.

Tucker, M., Lewis, D., Foster, P., Lucky, F., Yerby, L., Hites, L., \& Higginbotham, J. (2016). Community-based participatory researchspeed dating: An innovative model for fostering collaborations between community leaders and academic researchers. Health Promotion Practice, 17, 775-780.

United States Census Bureau. One in five Americans live in rural areas. 2017. Retrieved May 5, 2021, https://www.census.gov/library/ stories/2017/08/rural-america.html.

Yaemsiri, S., Alfier, J. M., Moy, E., Rossen, L. M., Bastian, B., Bolin, J., Ferdinand, A. O., Callaghan, T., \& Heron, M. (2019). Healthy People 2020: Rural areas lag in achieving targets for major causes of death. Health Affairs, 38, 2027-2031.

Publisher's Note Springer Nature remains neutral with regard to jurisdictional claims in published maps and institutional affiliations. 